Cipango Cahiers d'études japonaises

16 | 2009

L'invention des « arts populaires » - Yanagi Sōetsu et le Mingei

\title{
Mouvement des Arts populaires et études folkloriques
}

The Folk Crafts Movement and Folklore Studies

\section{Damien Kunik}

\section{(2) OpenEdition}

Journals

\section{Édition électronique}

URL : https://journals.openedition.org/cipango/374

DOI : $10.4000 /$ cipango. 374

ISSN : 2260-7706

\section{Éditeur}

INALCO

\section{Édition imprimée}

Date de publication : 1 janvier 2009

Pagination : 89-104

ISSN : 1164-5857

\section{Référence électronique}

Damien Kunik, « Mouvement des Arts populaires et études folkloriques », Cipango [En ligne], 16 | 2009, mis en ligne le 18 novembre 2011, consulté le 30 juin 2021. URL : http://journals.openedition.org/ cipango/374 ; DOI : https://doi.org/10.4000/cipango.374

Ce document a été généré automatiquement le 30 juin 2021.

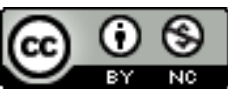

Cipango est mis à disposition selon les termes de la Licence Creative Commons Attribution - Pas d'Utilisation Commerciale 4.0 International. 


\title{
Mouvement des Arts populaires et études folkloriques
}

The Folk Crafts Movement and Folklore Studies

\author{
Damien Kunik
}

1 Lorsqu'en 1941 Yanagi Sōetsu (ou Muneyoshi) 柳宗悦 rédige un texte intitulé «Études sur les arts populaires et études folkloriques » à l'intention de la revue Kōgei 工藝 (Artisanat) $^{1}$, le mouvement pour les Arts populaires (Mingei undō 民藝運動) qu'il a largement contribué à créer est à son apogée ${ }^{2}$. L'Association pour les arts populaires (Nihon mingei kyōkai 日本民藝協会), organe directeur du mouvement fondé sept ans auparavant, enregistre son pic d'adhésions et réunit sous sa bannière plusieurs milliers de sympathisants. Les deux revues publiées par l'association, Artisanat et le Mensuel des Arts populaires (Gekkan Mingei 月刊民㙯) connaissent leur plus fort tirage. La Maison des arts populaires du Japon (Nihon Mingeikan 日本民藝館), l'un des fers de lance de l'activité du groupe, fête sa cinquième année d'existence dans le quartier de Komaba 駒 場 à Tōkyō. Yanagi, quant à lui, a déjà rédigé l'ensemble des textes qui constituent le corpus fondateur du courant ${ }^{3}$. Chez les membres du mouvement, le travail de promotion des théories esthétiques cède à cette période la place à un activisme engagé sur une scène nationale élargie. Yanagi s'implique ainsi dans un débat pour la sauvegarde du dialecte et de la culture des Ryūkyū. Yoshida Shōya 吉田璋也 (1898-1972) ou Shikiba Ryūzaburō 式場隆三郎 (1898-1965), deux figures clés de l'Association pour les arts populaires, investissent des efforts considérables en Mandchourie, en Corée ou à Taïwan ${ }^{4}$, où ils cherchent entre autres choses à étendre l'influence de l'Association.

2 La preuve la plus évidente de la réussite du mouvement se mesure à la pénétration de l'esthétique promue par les Arts populaires en de nombreux lieux de la culture urbaine. Depuis les années 1930 par exemple, les grands magasins, Mitsukoshi et Takashimaya en tête, organisent régulièrement des expositions temporaires pour promouvoir les objets valorisés par Yanagi et les siens. Quand on sait l'importance de ces institutions et leur influence sur les goûts des consommateurs, il semble presque certain que les Arts populaires sont alors plus largement perçus comme un mouvement d'art décoratif 
bourgeois que comme un courant intellectuel marginal ou un mouvement esthétique avant-gardiste.

3 Cependant, les objets d'artisanat rural japonais qui apparaissent dans les grands magasins, conçus comme des biens de consommation modernes, ne craignent pas d'affirmer leur couleur locale et populaire. Le style de ceux-ci est même plus précisément puisé dans des régions reculées de la nation, et jusqu'alors plutôt déconsidérées (San.in, Tōhoku), mais redécouvertes pour leur «haute teneur en japonité ». Ces objets sont enfin présentés comme la manifestation visible d'une théorie esthétique et sociale engendrée quelques années plus tôt par des personnes cherchant à revaloriser le patrimoine matériel national, en déclin face à l'industrialisation, la modernisation et l'occidentalisation du pays.

C'est peut-être pour ces raisons qu'il existe une confusion certaine entre les théories du mouvement des Arts populaires et celles d'un autre mouvement né durant la même période et qui touche à un sujet sensiblement similaire, celui des études folkloriques (minzokugaku 民俗学) de Yanagita Kunio 柳田國男 (1875-1962) auquel nous allons nous intéresser ici. Cette assimilation qui, hors des cercles de spécialistes, perdure encore aujourd'hui au Japon, n'est donc pas récente et trouve au contraire son origine durant les années d'émergence de ces deux approches, parfois semblables, parfois complémentaires, et parfois profondément antithétiques.

Nous ne pouvons nier la synchronie de l'émergence des deux mouvements, et l'influence d'une époque sur l'apparition d'un intérêt commun. N'oublions pas que d'autres contemporains de Yanagi et de Yanagita, Yamamoto Kanae 山本鼎 (1882-1946) dans le domaine des arts ou Miyazawa Kenji 宮沢賢治 (1896-1933) dans celui des lettres, se prennent eux aussi de passion pour les questions de revalorisation du patrimoine populaire et rural. Ce goût pour le sujet dans la première moitié $d u x^{e}$ siècle japonais réunit donc a posteriori des acteurs qui ne se sont pas forcément côtoyés. L'utilisation d'un même idéogramme, min 民 (peuple, nation), nourrit en outre la confusion. Il y a enfin, chez Yanagi comme chez Yanagita, cette cause commune, cette volonté de lutter pour la préservation d'un patrimoine culturel et matériel régional, national et avant tout, asiatique.

6 Lorsqu'il rédige " Étude sur les arts populaires et études folkloriques ", petit essai d'une quinzaine de pages sur lequel nous nous pencherons ici, cette confusion, Yanagi en a pleinement conscience. C'est donc avec pertinence qu'il ressent la nécessité de composer un texte qui lui permette d'expliciter points communs et divergences entre son système théorique et celui de Yanagita. La nécessité est d'autant plus grande que 1941 marque une nouvelle étape dans la mobilisation de tous à l'effort de guerre. Les arts et les sciences sont également sollicités, plus encore que dans la décennie précédente, et sont soumis à une réorganisation importante, à une centralisation d'activités qui pouvaient jusqu'alors exister de façon parallèle. Yanagi est, comme Yanagita, très certainement contraint de légitimer l'indépendance de son mouvement et des institutions qu'il a suscitées.

Dans le même temps, "Études sur les arts populaires et études folkloriques", qui cristallise cinq années de réflexions sur le rapport entre science et arts populaires, clôture un dialogue entrepris depuis 1936 entre Yanagi et plusieurs grandes figures du courant des études folkloriques japonaises, dont Shibusawa Keizō 渋沢敬三 (1896-1963), Miyamoto Tsuneichi 宮本常一 (1907-1981) et Yanagita Kunio lui-même ${ }^{5}$. 
8 À noter ici que les deux mouvements, qui perdurent à ce jour, n'ont plus jamais, après cette date, manifesté le désir ou le besoin de se rencontrer et qu'ainsi, s'il est tentant de rapprocher Yanagita et Yanagi pour leur travail de préservation du patrimoine culturel et matériel populaire, c'est en vérité un silence poli qui divise sympathisants des Arts populaires et folkloristes japonais.

9 Si donc il est risqué de vouloir associer trop rapidement les Arts populaires et le courant des études folkloriques, il paraît tout aussi réducteur de cantonner au seul domaine de l'histoire des arts décoratifs le mouvement de Yanagi, comme certains chercheurs occidentaux et japonais, et certains commissaires des récentes expositions sur le sujet, ont eu tendance à le faire ${ }^{6}$. Bien évidemment, à considérer la manière actuelle de donner une couleur exotique au terme mingei ${ }^{7}$, on pourrait penser que le mouvement des Arts populaires n'est pas plus proche des études folkloriques que la peinture naturaliste ne l'est de la biologie ou de la botanique. À Yanagi, une esthétique japonisante d'autrefois, à Yanagita, la science, en quelque sorte. Sans doute cette lecture des choses n'est-elle pas dénuée de tout fondement, quand on fait du mouvement des Arts populaires un courant artistique ancien. Mais il est important de dépasser cette lecture réductrice du mouvement, en prenant au sérieux les intentions de ses fondateurs, qui ont d'ailleurs été claires. Yanagi les pose, dès la première phrase de son texte ${ }^{8}$ :

Clarifier la nature des Arts populaires en tant que discipline scientifique, voilà l'objectif de ce texte. Et pour entrer immédiatement dans le vif du sujet, il ne nous paraît pas inopportun de proposer une comparaison du mouvement des Arts populaires avec le courant des études folkloriques, car ces deux approches possèdent tant des similitudes que des positions divergentes. Tirer de la sorte des parallèles entre ces deux sciences est d'une part nécessaire, mais également chose enrichissante. Il est ainsi bénéfique de saisir que ces deux approches sont vouées à collaborer et à s'entraider à l'avenir.

Le plus remarquable point commun entre les études des arts populaires et les études folkloriques est que toutes deux placent au centre de leurs préoccupations la vie quotidienne du peuple.

10 Ainsi, si le mouvement des Arts populaires et celui des études folkloriques sont bien souvent étudiés par des spécialistes d'obédiences différentes pour des raisons que nous chercherons à élucider, et si le terme mingei est traditionnellement associé aux arts décoratifs japonais, il faut admettre que Yanagi lui-même a souhaité rapprocher son mouvement d'ambitions académiques telles que celles de Yanagita et de ses disciples. Ne serait-ce que de façon théorique, Yanagi aura là affirmé la volonté de promouvoir un savoir bien plus qu'une nouvelle forme esthétique.

11 Le présent article propose, à travers l'analyse détaillée du texte "Études sur les arts populaires et études folkloriques", de lever le voile sur un visage moins connu du mouvement, celui des interrogations et des remises en question théoriques, à cent lieues des réalisations célèbres des champions des Arts populaires que sont Kawai Kanjirō 河井寛次郎 (1890-1966), Hamada Shōji 濱田庄司 (1894-1978) ou Munakata Shikō 棟方志功 (1903-1975).

\section{Arts populaires (mingei) et outils (mingu)}

La première manifestation écrite d'une réflexion sur les points communs et les divergences entre le domaine des arts populaires et celui des études folkloriques est 
signée de la main de Shibusawa Keizō, dans un article rédigé en 1933 et intitulé «Le développement de l'Attic Museum $»^{9}$.

Petit-fils du célèbre économiste Shibusawa Eiichi 渋沢栄 一 (1840-1931), Shibusawa Keizō est avant tout connu aujourd'hui pour avoir repris la charge héritée de son illustre ancêtre. Son investissement personnel dans la finance, son poste de gouverneur de la Banque du Japon et de ministre de l'Économie dans l'immédiat après-guerre occultent souvent le fait qu'il est l'une des grandes figures des études folkloriques japonaises.

14 Dans le domaine scientifique qui est le sien, son nom n'est de toute évidence pas aussi célèbre que celui de Yanagita. La chose s'explique certainement par le fait que Shibusawa travaille essentiellement sur un aspect de l'étude du folklore japonais qui préoccupe peu le courant le plus orthodoxe: la collecte et l'analyse des objets du quotidien (jouets, outils, ex-voto, vaisselle). Quinze ans avant la création de la Maison des arts populaires, Shibusawa rendra public l'accès à ses collections par le biais d'un petit musée qu'il ouvre en 1921 dans les combles de sa maison de Mita (Tōkyō), et qu'il nomme, pour cette raison, l'Attic Museum アチック・ミュゼアム, soit littéralement, le «musée dans le grenier ». Le fait ne manquera pas d'inspirer Yanagi. Les collections du musée, disparu aujourd'hui, ont été pour l'essentiel acquises par le Musée national d'ethnologie (Kokuritsu minzokugaku hakubutsukan 国立民族学博物館), lors de la création de ce dernier à Ōsaka en $1974^{10}$.

Cet intérêt pour le patrimoine matériel japonais se retrouve également dans le nom qu'il donne à son domaine de recherche, «l'étude des outils populaires » (mingugaku 民 具学). Ceci explique donc très simplement que Shibusawa puisse s'intéresser aux travaux de Yanagi sur un domaine à priori sensiblement similaire, celui de l'artisanat japonais. Les deux hommes se rencontreront par deux fois ${ }^{11}$, lors d'une visite de Yanagi à l'Attic Museum d'abord, puis lors d'une enquête de terrain ${ }^{12}$ organisée conjointement par l'Association pour les arts populaires et quelques folkloristes, dont Shibusawa, au Festival des fleurs (hana matsuri 花祭) du canton de Shitara (Shitara-gun 設楽郡) dans le département d'Aichi.

Il peut donc être pertinent, pour opérer une première distinction entre le domaine des études folkloriques et celui du mouvement des Arts populaires, de s'intéresser à la spécificité de ces deux termes: mingei 民藝 (art/artisanat populaire) tel qu'il est employé par Yanagi, et mingu 民具 (outils populaires) tel qu'il est conçu par Shibusawa.

Lorsque l'on donne à ces objets [mingu] le nom de getemono ou de minge $\mathrm{i}^{13}$, l'on recherche avant tout à souligner la beauté individuelle de ceux-ci. À l'inverse, nous, à l'Attic Museum, nous les considérons comme partie intégrante de la vie quotidienne et nous essayons de révéler la nature de ceux qui les ont réalisés. En d'autres termes, les pièces présentées ici forment un tout, et laissent entrevoir l'âme de nos ancêtres. C'est dans celle-ci que nous percevons la beauté. ${ }^{14}$

Plusieurs choses doivent être relevées ici. En premier lieu, la logique voudrait que le terme mingu renvoie aux outils ( $g u$ 具) du peuple ( $\min$ 民), c'est-à-dire aux objets à vocation purement utilitaires, alors que le terme mingei ferait plutôt référence à un art (gei 藝) populaire, dont la dimension esthétique, même réduite à sa plus simple expression, doit être prise en compte. Cependant, si l'on en croit l'extrait donné cidessus, les termes mingu, getemono et mingei, puisqu'il semble possible de les intervertir, sont avant tout des étiquettes qui relèvent d'appréhensions différentes d'objets 
similaires. Ce constat contribue donc à relever une similitude fondamentale entre les deux approches : l'étude d'un champ commun.

Ces objets, auxquels membres du mouvement des Arts populaires et folkloristes accordent la même attention et la même valeur patrimoniale, ne jouent cependant pas le même rôle dans l'élaboration des théories respectives. Comme le précise Shibusawa, le mouvement des Arts populaires se préoccupe en premier lieu de souligner la beauté spécifique de chacun des objets de ce patrimoine. De son côté, le folkloriste n'a que faire de l'esthétique et cherche à travers eux à saisir la nature de la communauté japonaise par l'analyse de son patrimoine matériel.

19 Voilà donc présentée une première définition de ces deux approches que sont les études folkloriques et le mouvement des Arts populaires. La première s'intéresse aux mingu, aux objets dans leur dimension fonctionnelle et décrit la nature de ceux qui les utilisent, alors que la seconde se préoccupe de la valeur esthétique de ce même patrimoine.

\section{Les choses et les faits}

20 Cette première distinction entre Arts populaires et études folkloriques, qui ferait de Yanagi un observateur de la beauté et de Shibusawa un analyste de la nature du peuple japonais, si elle est pertinente, est cependant loin d'être suffisante pour résumer les propriétés respectives des deux mouvements. Shibusawa est en fait le représentant d'un aspect très marginal des études folkloriques. La description des manifestations culturelles populaires par le biais du patrimoine matériel est une méthode peu orthodoxe au sein de la discipline. Yanagita, qui laissera la plus forte empreinte dans le domaine, s'intéressera toujours beaucoup plus à l'étude du folklore par le biais des rites et des festivals religieux ou des traditions orales et du vocabulaire du monde rural japonais.

21 Peut-être ses rencontres avec Shibusawa font-elles prendre conscience de la chose à Yanagi. Le fait est que ce dernier proposera dans un article de 1936 intitulé « Les choses et les faits ${ }^{15}$, une seconde distinction entre les deux approches, qu'il précisera avec encore plus de concision et de pertinence dans «Études sur les arts populaires et études folkloriques ». Il suggère là que si les deux domaines se préoccupent en effet de culture populaire, de "vie quotidienne du peuple $»^{16}$, les Arts populaires s'intéressent aux objets et à l'artisanat japonais, qu'il nomme ici mono, les " choses concrètes ", alors que les études folkloriques sont elles plus tournées vers une analyse des phénomènes immatériels, sociaux, rituels ou religieux, les « faits » (koto).

Il est inévitable que les études des arts populaires prennent pour sujet les objets concrets, alors que dans le cas des études folkloriques, les objets servent plutôt de matériaux complémentaires. Il nous arrive notamment bien souvent de prendre des céramiques en exemple, alors que les folkloristes ne s'expriment que très peu au sujet de ces objets pourtant extrêmement courants dans la vie populaire japonaise. [...] À l'inverse, les études folkloriques se penchent beaucoup plus sur les rituels religieux ${ }^{17}$.

L'argument est pertinent et répond à une perception toujours actuelle de la nature des deux mouvements. Ainsi, si le mouvement de Yanagi s'illustre encore aujourd'hui par un intérêt évident pour le patrimoine matériel populaire, les études folkloriques se concentrent plutôt sur les pratiques japonaises et ce que celles-ci disent de la culture japonaise. 

angle ${ }^{18}$ :

On devrait pouvoir dire, à la lumière des travaux qui portent sur les outils populaires [民具 mingu], que les études folkloriques s'intéressent elles aussi aux choses concrètes [具体的な もの gutaiteki na mono]. Cependant, à y regarder de plus près, plutôt que pour parler des objets populaires eux-mêmes, ceux-ci sont collectés pour parler des événements [koto] qui les entourent. Plutôt que d'observer les choses [mono], les folkloristes regardent les faits qui entourent les choses. Plutôt que d'observer des objets populaires concrets, ils observent le monde abstrait qui entoure les outils populaires.

Tout en montrant que les deux approches diffèrent dans ce cas sur leur objet d'étude, Yanagi admet cependant que folkloristes et tenants de la méthode des Arts populaires étudient "des éléments qui finissent toujours par se mêler les uns aux autres ${ }^{19}$. Malgré tout, pour Yanagi, le mouvement des Arts populaires n'a pas la vocation d'être une science descriptive du patrimoine matériel. Il s'interroge au contraire sur la manière de soustraire l'objet au contexte de son utilisation. Le dessein de son approche est différent, car il ne s'agit pas de saisir l'objet dans son contexte factuel, mais de percevoir un élément qui transcende son milieu et son temps : la beauté, qui lui confère sa vraie valeur. Il l'explique de la sorte ${ }^{20}$ :

Prenons l'exemple suivant. Imaginons un instant que nous présentions à un client un bol à thé. Nous pourrons alors remarquer deux types de réactions. Certains diront: "Quel est le nom de ce bol ? D'où provient-il ? De quand date-t-il ? Comment s'appelle son créateur? "Ce type de client s'intéressera donc plus aux éléments qui entourent le bol qu'au bol lui-même. D'autres, au contraire, s'exclameront avec admiration: "C'est un bol magnifique!» ou encore: "Il est d'un goût remarquable!». Et comme c'est la valeur esthétique du bol qui attire leur ceil, ils ne s'intéresseront que dans un second temps aux événements qui entourent cet objet. D'un côté, il y a ceux qui jugent en misant tout sur le monde factuel. De l'autre, il y a ceux qui, en regardant directement les choses, recherchent la valeur véritable que possèdent ces choses, c'est-à-dire la beauté.

Ainsi, bien qu'ils se préoccupent d'un patrimoine matériel japonais concret, les Arts populaires s'intéressent surtout à la valeur esthétique de ce patrimoine. Ils s'éloignent donc des études folkloriques non seulement sur la question de l'objet d'étude, culture matérielle contre phénomènes culturels, mais également sur la question de la valeur intrinsèque de cet objet, et n'hésitent pas à extraire celui-ci de son milieu pour n'en garder que l'essence.

Cette question de la décontextualisation du patrimoine artisanal japonais est d'ailleurs toujours au centre de la «méthode des Arts populaires». Une simple visite à la Maison des arts populaires aujourd'hui confirme que les objets exposés ne sont classés ni chronologiquement ni géographiquement, mais plutôt par appartenance à des techniques artisanales indigènes: céramique, laque ou travail du bois par exemple. L'objet est donc extrait de son époque et de son lieu d'origine pour venir seulement témoigner de sa valeur esthétique et du génie de l'artisan anonyme qui lui a donné forme.

\section{Approche axiologique et approche descriptive}

Ce constat constitue l'argument principal du texte «Études sur les arts populaires et études folkloriques » et pour les raisons que nous allons expliquer ici, donne finalement un sens au terme mingeigaku, les « études sur les arts populaires » ${ }^{21}$. 
Pour comprendre la portée de ce terme, il est bon de relever que le texte emprunte considérablement au modèle théorique $\mathrm{du}$ philosophe néo-kantien Wilhelm Windelband (1848-1912), largement traduit au Japon à cette époque ${ }^{22}$, et à sa théorie de la complémentarité des jugements de faits et des jugements de valeurs. Dans son ouvrage Histoire et science de la nature (Geschichte und Naturwissenschaft), publié en 1894, Windelband affirme que l'opposition entre les sciences naturelles et les sciences de l'esprit ne porte en vérité pas sur l'objet d'étude, mais plutôt sur les méthodes employées par les unes et les autres pour en arriver à leurs conclusions. C'est exactement selon cette formule que Yanagi envisage de faire des Arts populaires une science qui, en adoptant sa propre méthode, peut offrir une crédibilité scientifique similaire à celle des études folkloriques. La distinction véritable entre son approche et celle de Yanagita s'articule autour du fait que si les études folkloriques sont une science descriptive, comme peuvent l'être la physique ou la biologie, le Mingei, bien plus qu'un simple courant esthétique, possède la forme d'une science axiologique, c'est-à-dire d'une science des valeurs (Wert), une forme d'outil éthique et normatif de la valeur du patrimoine ${ }^{23}$ :

Nous appelons sciences empiriques [経験学 keikengaku] les sciences qui décrivent la réalité, et sciences normatives [規範学 kihangaku] les sciences qui traitent de ce qui devrait être ${ }^{24}$. Les études folkloriques sont ainsi de la première catégorie, les études des arts populaires sont de la seconde.

Prenons un autre exemple. La botanique appartient au domaine du réel, elle désigne le monde de la réalité. À l'inverse, l'éthique manipule des concepts qui traitent d'un monde d'idéaux. Il existe une différence fondamentale entre les deux. La biologie se doit d'être une science descriptive, alors que l'éthique doit poursuivre l'établissement de normes. [...] La botanique n'est pas la science de la beauté des plantes. L'éthique, elle, n'est pas la science de l'homme, elle est la discipline qui traite des comportements humains justes. [...]

Le grand objectif de l'étude des arts populaires est l'établissement d'une norme dans la critique de la valeur.

Ainsi, si Yanagi considère effectivement que le matériau de base qui vient nourrir sa science est sensiblement le même que celui qui peut intéresser les folkloristes, il envisage d'utiliser celui-ci comme élément normatif pour la constitution d'une nouvelle esthétique nationale. Ce concept de «science normative » constitue donc la spécificité des études sur le mingei, et comme nous allons l'illustrer par l'exemple maintenant, il fera office de fondement dogmatique dans la mission de revalorisation du patrimoine matériel populaire japonais entrepris par le mouvement dans les années 1930-1940.

\section{D'une science normative aux rayons des magasins}

Le mouvement des Arts populaires est aujourd'hui bien trop souvent ramené à quelques noms dont l'importance est indiscutable certes (Kawai, Hamada, Yanagi), mais dont l'effort est peut-être le moins représentatif de ce qu'a pu être ce courant durant les années d'immédiat avant-guerre. Nous avons rappelé le nombre d'adhésions record à l'Association pour les arts populaires durant les années 1940. Si Yanagi restera toujours le théoricien du mouvement, si Kawai et Hamada seront toujours les héros des expositions consacrées au sujet, ce sont en vérité les milliers de sympathisants réunis sous la bannière de l'Association qui ont donné au mouvement son poids, son importance et sa capacité à imposer justement une norme esthétique nouvelle. 
31 Parmi ces sympathisants, nous en retiendrons un, totalement voué à la cause de Yanagi, et dont l'exemple pris parmi une multitude d'autres illustre à merveille l'application pragmatique des théories du mouvement. Yoshida Shōya 吉田璋也 (1898-1972), otorhino-laryngologiste originaire du département de Tottori, rencontre une première fois Yanagi en 1919. Séduit par le personnage, il consacre dès lors son temps libre à collectionner des objets populaires et à promouvoir la doctrine naissante du mingei, qui n'a à cette époque pas encore de nom. Lorsqu'il retourne, en 1931, dans sa ville natale de Tottori, il fait l'acquisition d'anciens entrepôts à grain qu'il transforme et restaure. En juin 1932, il ouvre là le premier musée consacré à l'artisanat populaire japonais ${ }^{25}$, ainsi qu'un magasin qui occupera dès lors la majeure partie de son temps.

Yoshida souhaite d'abord redonner vie à l'artisanat de sa région, durement touchée par la crise économique de ces années. Il contacte donc plusieurs artisans de Tottori et leur assure l'écoulement de leur marchandise par le biais de son échoppe, qu'il nomme Takumi たくみ (Métier). Préoccupé également par la disparition de certaines techniques artisanales, il garantit la vente des objets produits par des artisans qui choisissent de revenir à celles-ci ${ }^{26}$. Yanagi, ravi par la détermination de Yoshida, lui consacrera plusieurs articles dans la revue Kōgei.

Le succès semble au rendez-vous. Les recettes dégagées par la production céramique permettent d'investir dans la production textile. Yoshida fait s'installer chez lui des tisserandes auxquelles il présente des exemples issus de sa propre collection ${ }^{27}$. Les techniques retrouvées, ces tisserandes en formeront d'autres et, par effet exponentiel, la production textile permettra d'investir dans la menuiserie, le laque, puis le métal, la vannerie, le papier, et enfin la production de pierres à encre.

La région de Tottori finit par ne plus suffire à écouler la production croissante des artisans associés au magasin. C'est ainsi qu'en décembre 1933 une annexe de Takumi est ouverte à Ginza, en plein cœur de Tōkyō. Celle-ci continue aujourd'hui, selon la même formule, à soumettre des commandes à des artisans japonais qui s'inspirent d'objets ou de savoir-faire hérités du passé, et d'écouler leur production pour assurer un soutien financier à l'Association tout en faisant survivre des métiers manuels représentatifs, en théorie du moins, d'un patrimoine national ${ }^{28}$.

Une visite au magasin Takumi de Ginza possède cela d'intéressant que les biens qui y sont exposés diffèrent considérablement des pièces présentées dans les expositions consacrées au Mingei. Si nous avons tous en tête quelques vases de Kawai, les bols de Hamada ou certaines grandes coupes de Bernard Leach (1887-1979), ce sont des fourchettes, des cravates, des coussins et des chapeaux qui s'empilent sur les rayonnages de l'échoppe de Ginza. Et que l'on ne s'y trompe pas, ce sont ces modestes objets consommables qui ont certainement mené le mouvement des Arts populaires vers sa réussite dans les années 1940. Il n'y a rien de plus faux que de croire que le Mingei est fait seulement de théories et de pièces de musée. Sans l'adhésion d'un public de consommateurs se pressant en masse chez Takumi ou au grand magasin Takashimaya, le dogme esthétique fondé par Yanagi et ceux qui l'entourent n'aurait certainement pas dépassé le cercle des intellectuels proches du personnage. La traduction de l'entretien entre Yanagi et Yanagita donnée dans ce dossier est à ce titre extrêmement parlante, puisqu'elle illustre les deux préoccupations majeures du mouvement dans les années 1940 : la définition de sa nature d'une part (légitimer son existence), et la promotion de ses produits de l'autre (assurer sa survivance). 
36 C'est ainsi que se réalise finalement le concept d'approche normative vu précédemment. Derrière ces objets vendus chez Takumi ou lors des expositions organisées dans quelques grands magasins, il existe en filigrane cette théorie selon laquelle le patrimoine matériel populaire japonais doit servir de norme pour produire, selon les critères de beauté édictés par Yanagi durant près de vingt ans, des biens de consommation permettant à leur tour de répandre ces critères dans l'ensemble des strates de la population japonaise ${ }^{29}$. C'est donc très certainement sur ce point que se distinguent finalement le plus clairement études folkloriques et mouvement des Arts populaires. Si la première de ces deux approches est, dans les termes que l'on connaît, une science descriptive, la seconde aura cherché à promouvoir un idéal esthétique qui puise certes dans le même vivier, mais qui veut susciter une nouvelle production, commercialisable. Il ne fait pas de doute que c'est pour cette raison que folkloristes et sympathisants des Arts populaires n'ont plus jamais ressenti le besoin, après 1941, de tisser des liens ou d'œuvrer ensemble à l'élaboration d'une science commune, leurs objectifs étant trop divergents.

\section{Conclusion}

Cet article avait l'ambition de prendre au sérieux la position « scientifique » de Yanagi. Nous avons pour cela analysé les rapports entre le mouvement des Arts populaires et le courant des études folkloriques, pour chercher à comprendre points communs et divergents entre ces deux approches inspirées par un patrimoine commun et un intérêt grandissant pour le "peuple» à l'époque de leur élaboration. Si toutes deux s'intéressent au même moment aux productions, culturelles ou matérielles, des classes populaires - et plus précisément, rurales - de la société japonaise, nous avons pu déterminer que c'est la valeur intrinsèque attribuée à l'objet d'étude qui établit la distinction fondamentale entre ces deux courants. Les études folkloriques cherchent, par un travail de compilation des pratiques populaires, à saisir et à déterminer la nature du peuple de l'archipel. Le mouvement des Arts populaires a, de son côté, pour ambition de réévaluer la beauté de la production matérielle de ce même peuple, et de poser cette manifestation du Beau comme une norme nouvelle. Ceci nous amène donc à rappeler le propos de Yanagi qui propose de comprendre les études folkloriques comme une science empirique et les études des arts populaires comme une science normative.

Nous avons présenté ici deux aspects du Mingei, parmi tant d'autres, qui ne trouvent encore que trop rarement leur place dans les ouvrages consacrés à la question. En premier lieu, nous l'avons dit, les Arts populaires se pensent comme une science, et s'appliquent donc à établir un système réfléchi et normé dont la structure n'a pas encore été analysée à ce jour. Ensuite, les Arts populaires se conçoivent comme un mouvement commerçant. Cette dimension, pourtant affirmée au magasin Takumi ou dans la boutique de la Maison des arts populaires de Tōkyō, semble cependant très régulièrement marginalisée dans les discours sur le sujet. Le mouvement a pourtant la réelle volonté de redonner vie à la production artisanale des régions du Japon, selon une norme esthétique redéfinie, et s'engage activement à chercher des débouchés commerciaux à sa production de chapeaux, de coussins ou de pantoufles, au Japon comme à l'étranger.

39 La volonté d'établissement d'une nouvelle norme de beauté et les préoccupations de viabilité économique d'une nouvelle production artisanale constituent finalement deux 
éléments pertinents pour distinguer le mouvement des Arts populaires du courant des études folkloriques. Cependant, il est tentant à ce stade de prendre ces éléments à contre-pied et de réévaluer la nature des études folkloriques, observées cette fois-ci à travers le prisme du Mingei. Si nous acceptons que le mouvement des Arts populaires ait tout à la fois établi une norme et des produits de consommation, ne pourrait-on pas considérer également que les études folkloriques ont, elles aussi, constitué un système normatif définissant ce que devait être la "tradition» japonaise en produisant des formes culturelles idéalisées d'un passé pensé comme «juste »?

La question ne concerne plus directement le mouvement des Arts populaires, mais montre cependant combien une étude approfondie du sujet mériterait d'être menée et pourrait venir enrichir quantité de propos connexes. Et à notre grand regret, l'approche consensuelle offerte par la plupart des expositions sur le Mingei fait oublier plusieurs aspects essentiels de la nature de celui-ci, qui pourraient à leur tour offrir une lecture nouvelle de l'émergence des sciences humaines et sociales dans le Japon de la première moitié du vingtième siècle.

\section{NOTES}

1. «Mingeigaku to minzokugaku » 民藝學と民俗學 (1941), repris in Yanagi Sōetsu zenshū (YSZ) 柳 宗悦全集 (Euvres complètes de Yanagi Sōetsu), Tōkyō, Chikuma shobō, 22 vol., 1981, vol.9, p. $272-287$.

2. Le terme mingei 民芸 ou 民藝 est aujourd'hui d'usage relativement courant au Japon. Écrit avec l'idéogramme 芸, il évoque, par abus de langage, un artisanat populaire régional vendu dans les boutiques de souvenirs locaux. Pour cette raison, les spécialistes japonais du sujet préfèrent l'usage de l'ancien idéogramme 藝 (la graphie du caractère du temps de Yanagi) pour renvoyer au mouvement des Arts populaires et marquer clairement la distinction. Nous respectons ici cet usage.

3. Notamment, «Getemono no bi »下手ものの美 (La beauté des objets du quotidien, 1926), YSZ, op.cit., vol. 8, p. 3-14; “Kōgei no michi»工藝の道 (La voie de l'artisanat, 1928), Tōkyō, Kōdansha, coll. « Kōdan gakujutsu bunko », 2005.

4. Voir à ce sujet le chapitre "Renovating Greater East Asia", Kim Brandt, Kingdom of Beauty. Mingei and the Politics of Folk Art in Imperial Japan, Durham, Duke University Press, 2007, pp. 173-222.

5. Voir notre traduction dans ce dossier, http://cipango.revues.org/355.

6. Avec l'exposition L'esprit mingei au Japon du Musée du quai Branly à Paris, du 30 septembre 2008 au 11 janvier 2009, l'exposition Arts \& Crafts from Morris to Mingei au Musée d'art de la ville de Tōkyō (Tōkyō-to bijutsukan 東京都美術館), du 24 janvier au 5 avril 2009, et avant elles l'exposition International Arts \& Crafts du Victoria \& Albert Museum de Londres, du 17 mars au 24 juillet 2005.

7. Voir l'article de Coralie Castel dans ce même dossier, http://cipango.revues.org/389.

8. «Études sur les arts populaires et études folkloriques ", op. cit., p. 272, souligné par nous.

9. Achikku no seichō アチックの成長 (Le développement de l'Attic Museum) in Miyamoto Tsuneichi 宮本常一, Nihon minzokugaku taikei 3: Shibusawa Keizō 日本民俗学大系 3 ・渋沢敬三 (Collection des études folkloriques japonaises 3 : Shibusawa Keizō), Tōkyō, Kōdansha, 1978. 
10. Yoshida Kenji, "Tōhaku and Minpaku within the History of Modern Japanese Civilization: Museum Collections in Modern Japan" Senri Ethnological Studies, ${ }^{\circ} 54$, Osaka, National Museum of Ethnology, 2000, pp. 77-102, ici pp. 91-92.

11. Kanetani Miwa 金谷美和, «Bunka no shōhi: Nihon mingei undō no tenji o megutte » 文化の 消費一日本民藝運動の展示をめぐって (Consommer la culture. Au sujet des expositions du mouvement des Arts populaires japonais), Jinbun gakuhō 人文学報, $n^{\circ} 77$, Kyōto, Kyōto daigaku jinbunkagaku kenkyūjo, 1996, pp. 63-97, ici p. 80.

12. L'événement est relaté dans l'article «"Zazechi” no koto»「ざぜち」のこと (Au sujet des papiers découpés « zazechi »), YSZ, op. cit., vol. 11, pp. 424-432.

13. Jusqu'aux années 1940, les deux termes étaient utilisés indifféremment par Yanagi.

14. Achikku no seichō, op. cit., p. 168.

15. «"Mono" to "koto"»「もの」と「こと」(Les « choses » et les «faits ", 1936), YSZ, vol. 9, p. $170-181$.

16. « Mingeigaku to minzokugaku », op. cit., p. 272.

17. Ibid., p. 279.

18. Ibid., p. 279.

19. Ibid., p. 278.

20. Ibid., p. 278.

21. Nous trouverions un certain plaisir également à rendre mingeigaku par un bricolage du type « artisanologie populaire». Si cette seconde traduction ne possède évidemment aucun sérieux, elle rend bien l'étrangeté du terme.

22. Plus d'une douzaine d'ouvrages traduits entre 1902 et 1941.

23. « Mingeigaku to minzokugaku », op. cit., pp. 274 et 276.

24. Cette distinction entre ce qui « est", le Sein, et ce qui "devrait être ", le Sollen, est bien caractéristique de la pensée de Windelband.

25. Ogyū Shinzō 尾久彰三 (dir.), Yanagi Muneyoshi no sekai. «Mingei» no hakken to sono shisō 柳宗 悦の世界一『民藝』の発見とその思想, (L'univers de Yanagi Muneyoshi. Sa découverte des « arts populaires » et sa pensée), Tōkyō, Bessatsu Taiyō, Heibonsha, 2006, p. 171.

26. Kikuchi Yuko, Japanese Modernisation and Mingei Theory: Cultural Nationalism and Oriental Orientalism, New York, Routledge Curzon, 2004, p. 74.

27. Kim Brandt, op. cit., p. 95.

28. http://www.ginza-takumi.co.jp/top.htm

29. Quoique, dans les faits, la production d'un nouvel artisanat populaire ne soit accessible qu'aux classes relativement aisées de la population urbaine.

\section{RÉSUMÉS}

À la même période que le mingei de Yanagi apparaît le mouvement des études folkloriques (minzokugaku) de Yanagita Kunio. Souvent associés, les deux entretiennent toutefois nombre de différences.

At about the same time as mingei appeared Yanagita Kunio's Folkloristics (minzokugaku). Often confused, the two differ in many ways. 
INDEX

Thèmes : anthropologie, ethnologie

キーワード : minshū geijutsu 民䍃芸術, taishū bunka 大䍃文化, mingu 民具, mingei undō 民芸 運動, Yanagi Sōetsu 柳宗悦 (1889-1961), minzoku 民俗, minzokugaku 民俗学, Shibusawa Keizō 渋沢敬三 (1896-1963), jinruigaku 人類学, minzokugaku 民族学, Shōwa jidai 昭和時代

(1923-1945), Shōwa jidai 昭和時代 (1945-1989)

Keywords : Anthropology, Ethnology, Folk Art, Material Culture, Mingei, Popular Culture, mingu, Utensils, Yanagi Sōetsu (1889-1961)

Mots-clés : arts populaires, culture populaire, outils, mingei - mouvement artistique, Yanagi Sōetsu (1889-1961), folklore, études folkloriques

Index chronologique : Shōwa (1923-1945), Shōwa (1945-1989) 